\title{
Education versus screening: the use of capacity to consent tools in psychiatric genomics
}

\author{
Camillia Kong (1) , ${ }^{1}$ Mehret Efrem, ${ }^{2}$ Megan Campbell ${ }^{3}$
}

${ }^{1}$ School of Law, Birkbeck University of London Institute for Criminal Policy Research, School of Law, London, UK ${ }^{2}$ Department of Psychiatry, University of Oxford, Oxford, UK ${ }^{3}$ Department of Psychiatry and Mental Health, Faculty of Health Sciences, University of Cape Town, Cape Town, South Africa

\section{Correspondence to}

Dr Camillia Kong,Birkbeck University of London Institute for Criminal Policy Research, London, UK;

camillia.kong@bbk.ac.uk

Received 4 February 2019 Revised 30 July 2019 Accepted 1 August 2019 Published Online First 28 September 2019
Check for updates

(C) Author(s) (or their employer(s)) 2020. No commercial re-use. See rights and permissions. Published by BMJ.

To cite: Kong C, Efrem M, Campbell M. J Med Ethics 2020:46:137-143.

\section{ABSTRACT}

Informed consent procedures for participation in psychiatric genomics research among individuals with mental disorder and intellectual disability can often be unclear, particularly because the underlying ethos guiding consent tools reflects a core ethical tension between safeguarding and inclusion. This tension reflects important debates around the function of consent tools, as well as the contested legitimacy of decision-making capacity thresholds to screen potentially vulnerable participants. Drawing on human rights, person-centred psychiatry and supported decisionmaking, this paper problematises the use of consent procedures as screening tools in psychiatric genomics studies, particularly as increasing normative emphasis has shifted towards the empowerment and participation of those with mental disorder and intellectual disabilities. We expound on core aspects of supported decisionmaking, such as relational autonomy and hermeneutic competence, to orient consent procedures towards a more educative, participatory framework that is better aligned with developments in disability studies. The paper concludes with an acknowledgement of the pragmatic and substantive challenges in adopting this framework in psychiatric genomics studies if this participatory ethos towards persons with mental disorder and intellectual disability is to be fully realised.

\section{INTRODUCTION}

Informed consent in psychiatric genomics studies in the global context has proven to be a highly complex ethical issue to date. Challenges in lowmiddle-income countries related to cultural and linguistic translation are already well documented in genomics studies more generally, including the influence of low literacy levels, unfamiliarity with research protocols, lack of locally relevant terminology and therapeutic misconceptions on the quality of understanding of informed consent materials. ${ }^{1-3}$ Psychiatric genomics studies examine conditions typically classified as mental disorders (eg, schizophrenia, bipolar disorder, depression) as well as intellectual disabilities (eg, autism spectrum disorders). Cognitive impairment among participants of psychiatric genomics studies, combined with the often complex scientific terminology involved in the research, further complicate the purpose and function of informed consent procedures.

As a core pillar of ethical research practice, standard accounts of informed consent focus on safeguarding potentially vulnerable study participants from exploitation and harm. This safeguarding ethos has become particularly entrenched in consent procedures involving persons with intellectual disability, whereby tests of functional cognitive abilities (eg, understanding, appreciation and reasoning) serve as thresholds that individuals must meet in order to demonstrate decisional capacity to consent. Two well-established examples include the MacArthur Competence Assessment Tool (MacCAT-R) for clinical research ${ }^{4}$ and the briefer screening tool, the University of California, San Diego Brief Assessment of Capacity to Consent tool (UBACC). ${ }^{5}$ These informed consent tools are often used to screen to exclude potentially vulnerable participants who are unable to demonstrate sufficient decisional capacity to give informed consent.

This paper argues that an ethos of safeguarding could lead to the overprotection of participants considered particularly vulnerable due to cognitive impairment in the context of psychiatric genomics research. It is not our intent to probe the meaning or markers of consent, nor broader ethical issues specific to genomics research (such as questions around return of genetic information). Rather, we suggest that psychiatric genomics research clearly distills two problems, one specific to these studies in particular - that is, appropriate application of consent screening tools - and the other relevant to research involving individuals with mental disorders and intellectual disabilities more generally - that is, the ethical tension between appropriate safeguarding and protection on the one hand and permissible risk on the other.

First, there is prevailing uncertainty on the ground regarding the appropriate application of standard tools of consent in psychiatric genomics research among recruiters and those charged with designing research protocols. The practical reality is that psychiatric genomics studies involve complex scientific concepts and research study elements which persons with cognitive challenges may struggle to understand, thereby worsening power inequalities, translational issues and educational disparities already present in certain socio-cultural contexts. ${ }^{36}$ The traditional process of screening (to gauge understanding, appreciation, reasoning and so on) quickly excludes potential (more vulnerable) participants who come from genomically under-represented groups while an educative orientation can promote decisional capacity and understanding, enhancing the inclusivity of the research. For example, the adaptation of the UBACC for recruiting Xhosa people in a South African psychiatric genomics study highlights the legitimate role and purpose of iterative learning in the consenting process. $^{3}$

Second, the conflicting orientations in consent procedures-on display in psychiatric genomics studies-reveals broader difficulties in achieving an ethically grounded balance between appropriate 
safeguarding and protection on the one hand and permissible risk on the other, in ways that promote equal treatment and participation in research involving those with intellectual disability and mental disorder. A more participatory ethos in disability studies and person-centred psychiatry challenges the standard safeguarding orientation in informed consent. Inspired by the disability rights movement, principles of self-advocacy, individual autonomy, and reasonable accommodations have gained prominence to support the equal participation of persons with impairments in all domains of human life-including the ability to participate in research. For example, Article 31 of the United Nations Convention on the Rights of Persons with Disabilities (CRPD) stipulates how research and data should be used 'to identify and address the barriers faced by persons with disabilities in exercising their rights' and 'ensure their accessibility to persons with disabilities and others ${ }^{778}$. Meanwhile, the person-centred psychiatry movement aims to empower patients to be active participants in their own healthcare, with an emphasis on holistic healthcare that includes ill and positive health experiences within a combined biological, psychological, social, cultural and spiritual framework. ${ }^{9}$

Together this prism of human rights and the person-centred movement suggests the need for critical scrutiny of the default safeguarding ethos in research involving persons with mental disorders and intellectual disabilities. It is not our purpose to examine the clinical details and implications of specific conditions in consent procedures, but rather the consent procedures of psychiatric genomics studies more generally in light of the changing legal and ethical landscape towards persons with cognitive impairments of some kind. Importantly, the CRPD does not distinguish between different conditions in the rights, entitlements and obligations owed to people with disabilities (which encompasses mental disorder, physical and intellectual disability). For this reason, we use the term 'mental disorder and intellectual disability' throughout the paper.

By implication, researchers may have an ethical obligation to promote as many possible voices of people with mental disorder and intellectual disability, with an emphasis on the need for research that is inclusive and participatory. Such individuals are to be treated as co-producers of knowledge which promotes their equal treatment and respect. ${ }^{10} 11$ The implication of this normative reorientation is that informed consent ought to function with a similar inclusive ethos towards potential research participants, using mechanisms that support improved understanding of the research process and promote meaningful engagement with consent materials.

As welcome as this participatory framework is, however, it comes with a number of pragmatic challenges, including the costs of time and staff resources in implementation. Its implications for the future research modelling of psychiatric genomics studies also require some careful exploration: documentary/ qualitative methodologies which emphasise the subjective experiences and perspectives are often upheld as paradigmatic models for encouraging the participation and voices of persons with intellectual disability and mental disorder; yet, such methodologies are often absent in genomics studies. To fully realise the participatory ethos may demand some critical examination of the normative intent of and methods used in psychiatric genomics research.

\section{Human rights, person-centred psychiatry and universal legal capacity}

The growing prominence of a human rights perspective on disability has led to important changes in our attitudes and policies towards persons with mental disorder and intellectual disability. Aspirations to realise the equal treatment and respect for such persons are encapsulated in the CRPD and its articulation of widespread positive supports, obligations and reasonable accommodations that are needed to facilitate equal participation in society.

The human rights prism to disability and mental health represents a significant shift in moral emphasis in three ways. First, safeguarding and welfarist considerations which have traditionally dominated the treatment of persons with intellectual disability have given way to a participatory ethos and autonomy focus, premised on 'the dignity of risk' of individuals and their right to potentially fail, to learn from failure, to make choices that might be deemed risky or imprudent, regardless of varying levels of cognitive functioning. ${ }^{12}$ Putting persons with intellectual disability at the centre of decisions about their care, treatment and research participation means that unwise decisions (ie, decisions that may seem irrational or imprudent) are permissible without the threat of coercive third-party paternalistic interventions. (The concept of 'dignity of risk' raises important questions as to how we might define 'unwise' decisions and the threshold of 'reasonable risk', however, it remains beyond the scope of our paper to engage with this issue. ${ }^{1314}$ ) This participatory approach is further reflected in the person-centred psychiatry movement with the aim of empowering patients to take more ownership in their treatment and recovery. ${ }^{915}$ Implications for informed consent rest on how participants with disabilities are empowered around the 'dignity of risk'.

This question of empowerment is addressed in the second shift in moral emphasis: away from 'fixing' individuals with disability to the requisite supports that need to be in place within their societal and environmental context. Embedded within this human rights framework is a commitment to the social model of disability which attributes disability to environmental and structural barriers that hinder equal participation with others. ${ }^{1116}$ Impairments of the mind and body, on the other hand, are presumed to be value-neutral aspects of the person which require positive accommodation. This perspective again aligns well with the person-centred psychiatry movement with its emphasis on a holistic framework for understanding psychiatric illness from a combined biological, psychological, social, cultural and spiritual perspective. In short, the alleviation of disability demands societal, environmental and structural change, not fixating on what is 'wrong' with persons with disability.

The third and most fundamental shift in moral emphasis rests in the notion of universal legal capacity which adopts a critical, if not altogether sceptical stance towards tests of 'mental competence' or 'mental capacity' that determine whether or not persons can make decisions about their care, treatment, and potentially, research participation. The most extreme iterations of this position-such as the CRPD Committee's General Comment on Article 12-posit that the legal status, entitlements and rights of persons are disconnected entirely from mental capacity, and indeed, legislative regimes which claim legal capacity is contingent on meeting a threshold of decision-making competence are fundamentally discriminatory. ${ }^{17}$ The Committee therefore recommends the complete abolishment of mental capacity assessments of any kind. Though not mentioned explicitly, the logic of the Committee's stance would suggest its application also to research contexts involving persons with intellectual disabilities.

There are many rejoinders to the Committee's General Comment call to eliminate mental capacity assessments-not least on grounds of its philosophical, practical and ethical implications on the domains of mental healthcare, social care and 
law. ${ }^{18-20}$ We lack the space to engage in any depth with these important debates, nor are we positing that capacity thresholds have no place in research practices, particularly given the practical reality of cases of individuals with profound cognitive and communicative difficulties. The point to emphasise is that one need not go so far as the Committee's General Comment to recognise the important normative shift that universal legal capacity represents in its challenge to questionable assumptions within standard tests of mental competence (such as a preoccupation on internal cognitive processes and the putative justification of substituted decision-making regimes). That this human rights framework towards disability is gaining traction is apparent with the ratification of domestic legislation in line with the CRPD, with many countries using the framework to underpin research protocols. ${ }^{1121}$ Nonetheless, how consent tools should be applied and used in light of the participatory ethos remains relatively unexplored thus far, aside from an emphasis on participatory technologies and methodologies (such as the use of photography and voice diaries). ${ }^{21-26}$

Yet, these shifts in moral emphasis have profound implications on the function and role of consent tools, such as the need for a more nuanced and complex normative orientation: the safeguarding orientation of standard consent tools is no longer assumed to be appropriate in the first instance, given the importance of allowing persons with intellectual disabilities a degree of 'risk' in making their decisions and the role of such persons in co-producing knowledge about their lives and experiences. Current thinking about informed consent as a construct that can be screened and tested for, rather than promoted through iterative learning becomes unsustainable, given that the concept of decisional capacity as a binary, threshold concept may no longer be warranted. A critical stance towards the concept of decisional capacity need not entail the denial of the concept altogether, but could lead to understanding it more as a spectrum that is facilitated through iterative learning, dialogue, creative communicative tools and relational support. ${ }^{27}$

This suggests that consent tools used to screen, identify and exclude potential participants who are unable to meet criteria of decisional capacity could run counter to the inclusive and participatory ethos at the heart of the human rights approach to intellectual disability and, indeed, are potentially discriminatory towards persons with varying cognitive abilities. This is an important consideration that challenges current thinking about informed consent as a construct that can be screened and tested for, rather than promoted through iterative learning. At their most extreme, such screening tools could be perceived by recruiters and potential research participants as a 'test', aggravating anxiety around performance and abilities. More importantly, this singular focus on the individual's performance and abilities obscures a vital half of the consenting process-namely the supportive efforts of others and the environment around the individual (such as study recruiters) to explain and support. One need not be wholeheartedly committed to the social model of disability to recognise the importance of environmental, societal and relational factors in contributing to the exclusion and inequality of persons with cognitive impairments. Indeed, taking seriously the positive supports and reasonable accommodations required by those around persons with intellectual disability and mental disorder draws critical attention to how consent tools are delivered to potential participants-specifically the nature and quality of communication.

Further ethical exploration is needed as to whether consent tools in psychiatric genomics research should adopt the orientation of screening to exclude more vulnerable participants who struggle with traditional consent procedures as opposed to providing mechanisms that support improved understanding of the research process for such participants in order to be more inclusive. This is particularly important because of prevailing uncertainty on the ground regarding the appropriate application of standard tools of consent in psychiatric genomics studies.

\section{SUPPORTED DECISION-MAKING}

Our suggestion is that there is an ethical case for consent tools and procedures to be used as educational tools to help guide prospective participants through the relevant content of the study, and therefore help facilitate and support their ability to make a decision about whether or not to participate. This educative dimension is especially important to help promote comprehension of complex concepts and research information within psychiatric genomics studies. In this way, informed consent materials as educational tools provide opportunities to empower persons of different cognitive abilities to act as co-producers of knowledge about their experiences and conditions. Underlying this participatory ethos is a commitment to supported decisionmaking, an increasingly important legal, policy and theoretical framework that recognises the importance of enabling relationships and communicative mechanisms to foster decision-making capacity.

Supported decision-making, according to Gooding, 'refers to processes whereby a person is provided with support, if he or she so choose, to give expression to their wishes and preferences regarding a particular decision concerning him or herself' (Gooding, p.434). ${ }^{12}$ The supported decision-making paradigm fundamentally questions the individualist assumptions at the heart of functional measures commonly used in standard informed consent tools, such as the MacCAT-R. The core pillars of this model (understanding, reasoning, appreciation and communication) suggest that individuals can be presented with relevant information and it is up to them to process it, deliberate about its potential harms and benefits, and communicate a decision. Capacity is viewed as a placeholder for autonomy, where autonomy concerns the individual and his or her choices, expressed through cognitive abilities (internal intellectual skills and abilities of reasoning). This paints a picture of the ideal autonomous agent as a bounded, self-sufficient, independent and rational mind. Standard consent tools designed as screening tools to exclude those who are not able to meet these criteria are effectively determining the extent to which individuals and their cognitive skills meet an acceptable threshold which approximates this ideal.

By contrast, supported decision-making takes as its point of departure a model of relational autonomy which posits that individuals are interdependent beings, embedded within relational and social contexts that can fundamentally foster or diminish their ability to make decisions. Much less an achievement of isolated selves and bounded minds, the skills of autonomy are developed in relationship, through dialogue, enabling narratives and facilitating tools. Dependence, impairment, and support need not imply one lacks the capacity to make decisions. A relational model of autonomy takes a more holistic view of the self, as situated in and responsive to certain interpersonal conditions that can be crucial in the expression of skills of understanding, deliberating, and reasoning. ${ }^{27}$

This account of autonomy is important in the context of assessing capacity of those with mental disorder and intellectual disability for a couple of reasons. First, moving beyond the bounded self as the locus of informed consent suggests 
an inclusive picture more accommodating of diverse minds and bodies. ${ }^{27}{ }^{28}$ Instead of a disembodied picture of a person's mind and cognitive processes as functioning independently of emotion, perception and embodiment, what emerges instead is a picture of embodied selves, where non-cognitive processes and ways of interacting are just as significant as the mind in determining how skilfully a person navigates or copes with their environment, or understands certain decisions. Philosophers speak of absorbed coping, for example, to denote the pre-cognitive, perceptual skills involved in everyday bodily engagement with one's situation and environment. ${ }^{29}$ Behaviours that would seem to denote unthinking or lack of reflection may in fact be skilful ways of coping with changes in one's environment or responding to implicit cues in the relational context. For instance, the sudden pacing of an individual during the consent process may seem inexplicable in the first instance, but from a perspective of absorbed coping, be an understandable and skilful response to an unfamiliar environment, or perceptions of being 'tested' and pressures to answer correctly about relevant information.

Second, in recognising that autonomy and capacity can be consistent with support and relationality, the interpersonal conditions of enablement becomes the normative focus. ${ }^{27} \mathrm{In}$ other words, by recognising that all of us-whether or not we have a disorder or intellectual disability-require supportive contexts to help promote and enable us to express our authentic, autonomous selves, the focus becomes less so on the individual herself and her cognitive and intellectual competencies, and more so on the certain qualities within the relational, social and intersubjective context. Socialisation affects individuals and the ways in which they understand and express themselves, and autonomy will be exercised within intersubjective contexts; individuals engage with these socialising forces when forming values, preferences, and expressing decisions. This is especially salient in different cultural contexts, such as in African thought and practice, where a common core in this heterogeneous tradition is a communitarian ethos that regards individuals as fundamentally socially embedded and constituted ${ }^{30-33}$. Certain skills that are vital for a person's decision-making capacity will rely on those around her providing enabling support, for cultivating an environment that understands different ways of skilful bodily coping and communicating. Such skills can be evident in recruitment process. For example, in a recent South African schizophrenia genomics study, recruiters demonstrated significant differences in the number of participants they recruited. ${ }^{34}$ These differences were thought to reflect the powerful relational dynamics at play during the consent process and likely spoke to the divergent abilities of recruiters to effectively engage in discussions about complex genomic terms with their participants.

In short, conditions of dependency mean it is the responsibility of others to ensure that an individual's agency is supported, encouraged and enabled. Combined with the human rights lens demanding positive supports in place for persons with cognitive disabilities, this analysis corrects the current preoccupation with the cognitive capacities of individuals with mental disorder or intellectual disability in clinical research, moving instead towards the skills and capacities that are required by those around them. Researchers and recruiters are not necessarily passive, detached observers in the informed consent process. This therefore raises the question, what skills and competencies do interviewers and recruiters need to have if they are to enable and empower persons as potential participants with cognitive challenges? We turn to this question in the following section.

\section{HERMENEUTIC COMPETENCE}

The term hermeneutic competence has been used to describe the interpretive and communicative skills and supportive practices by those responsible for the care, treatment and assessment of decision-making capacity of persons with mental disorder and intellectual disability. ${ }^{27}{ }^{28}$ Hermeneutics describes methods by which meaning and understanding is conferred to words, actions and practices. Fundamentally, we are always engaged in a process of interpretation, and the significance of our interpretive lens becomes especially important where there may be a gap in ways of experiencing and perceiving the world. Hermeneutical competence articulates the process of bridging this gap so that one adopts the right interpretive orientation and understands well without making incorrect assumptions. In other words, ' $[t]$ hese interpretive skills can help frame the way we approach persons with disabilities, so that on the one hand, we don't lose sight of their unique individuality, potential abilities, and vulnerabilities, and on the other hand, we take responsibility for our role in communicating in a manner that makes them feel heard, understood, and validated' $\left({ }^{28}\right.$, p.59).

Three skills are important in hermeneutic competence: first, attunement to impairment describes how persons recognise the particular perceptual and bodily challenges of an individual. ${ }^{27}$ Mental disorder or intellectual disability does not define the person or predetermine the person's decision-making capabilities. This is important to emphasise with respect to informed consent because of the considerable variation in quality of understanding we see across population groups, for example, in both high-middle and low-middle-income countries ${ }^{35}$ and in both those who have a severe psychiatric diagnosis and those who do not. ${ }^{3}$ Instead, attunement to impairment denotes the acceptance of the unique embodiment of individuals, the reflective awareness of ways in which certain cognitive, perceptual or bodily challenges might affect a person's manner of interacting and engaging with her environment, and the adaptation to meet these challenges in order to foster common understanding.

Second, recognition of the person involves the ability to see and respect other individuals as separate persons with their own unique perspective. ${ }^{27}$ This might seem counterintuitive to the emphasis on relationality and community, and may also seem culturally specific to Western rather than other non-Western cultures. However, this does not imply viewing individuals as atomistic beings. What we mean here can be better illustrated by the negative case-where persons fail to be recognised. In engaging with persons with mental disorder and intellectual disability, there is a tendency to objectify and assimilate individuals. When objectified, persons are treated as an object rather than subject with their own values, views and narratives, while assimilation occurs when it is presumed others can automatically speak or know on their behalf. In both, implicit biases and prejudices about the nature of their cognitive skills or about their diagnosis are allowed to predetermine their perspective and particular capacities. Ultimately, recognition of the person demands respecting their subjective perspective, approaching individuals with mental disorder or intellectual disability as individuals who hold discrete values and views.

Finally, open dialogue and humility are required in order to become critically aware of how one's prejudices and biases can intrude in interpretation while humility counters the assumption of one's superior knowledge. ${ }^{27}$ Preparing for open dialogue will demand the other two skills, and it also demands the acknowledgement that we all begin with prejudices of some kind. Prejudice in the Gadamerian hermeneutical tradition is literally 
'prejudgement'-it is the standpoint from which we view and engage with something. ${ }^{36}$ We all have a view from somewhere. But what marks the skills of a hermeneutically competent person is an awareness of these prejudgements and a concerted effort to remove the internal barriers to understanding other individuals with mental disorder and intellectual disability. This will involve constant dialectical engagement with our prejudices, so as to challenge one's beliefs about mental disorder, intellectual disability and cognitive capacity, and active efforts to cultivate a stance of dialogical openness towards the knowledge and narratives of those with cognitive challenges.

These skills are demanding but they are increasingly important in light of the human rights lens, a person-centred psychiatry approach, and the supported decision-making mechanisms that are required to realise this normative shift towards participation and empowerment in practice. Informed consent tools likewise need to be responsive to the normative shift towards persons with mental disorder and intellectual disability. Ultimately, such skills of hermeneutic competence are most consistent with a particular educative rather than screening orientation in the consenting process. When consent tools are used as a screen to exclude, the presumption remains that those recruiters are somehow removed from the intersubjective context, that the impersonal communication of relevant information is simply to test whether or not a person can retain and process it in a rational manner. Yet, strong evidence to the contrary highlights the influential role recruiters play in the consent process. ${ }^{3} 34$ One's interpretive stance may be potentially far less forgiving of challenges in remembering complex concepts or data, or more restrictive in terms of what counts as genuine understanding and appreciation of the research goals. However, once we recognise the vital role of support in enabling such persons in expressing their wishes and values, certain skills of dialogue, understanding and interpretation become necessary on the part of recruiters.

As an educative tool orientated towards inclusion, the ways in which interviewers and recruiters form a critical part of the dialogical exchange is acknowledged, more specifically their role in actively engaging, supporting and enabling the person's cognitive processing and choice-making abilities. Such tools are attuned to the areas where individuals may struggle with information as well as the barriers to understanding (eg, language, communication methods, etc), offering supports to enhance their decision-making ability.

For instance, consent tools to facilitate iterative learning can be vital in improving understanding, and appreciation, particularly where it involves complex psychiatric genomics concepts. ${ }^{3}$ Tools like the UBACC might be initially developed as a screening tool for decisional capacity. However, when used to identify research study elements participants struggle to comprehensively understand, so as to revisit and clarify these through iterative learning, the consenting approach immediately shifts focus from one of screening to exclude, to one of supporting and empowering participant understanding, thus making the process as inclusive as possible. The position of recruiters is altered accordingly: rather than administering a screening tool, they use the tool facilitatively to empower participants by encouraging a deeper understanding of the research study elements.

\section{IMPLICATIONS}

The relationality and support implied in this decision-making framework raises two issues: (i) practical, pragmatic issues around the pressures of recruiting; (ii) deeper substantive questions regarding the relevance of the participatory ethos in the context of psychiatric genomics research. We will discuss these in turn.

\section{Pragmatic implications}

An educative approach towards consent tools raises three important pragmatic issues. First, recruiters must develop a depth of knowledge of the research study, as well as the science and ethics underpinning it, in order to confidently apply consent tools to facilitate improved understanding. ${ }^{37}$ This is a resource-intensive process that requires continued training and quality control of the consent process to ensure that information is shared to participants in a fairly consistent way across recruiters. ${ }^{34}$

Second, an educative approach demands more recruitment time,${ }^{37}$ sometimes as much as $30-45$ min per participant to have the necessary discussions with recruiters. ${ }^{3}$ This time demand can place recruiters in a difficult position of having to balance the pressures of recruitment targets with ensuring that participants genuinely understand what they are consenting to. Even as screening tools, the consent process involving persons with intellectual disability and mental disorder can take additional time that recruiters can ill afford in light of the pressures to quickly recruit or reject potential participants. Yet, genuine implementation of the participatory ethos of supported decision-making suggests that the recruitment process involving individuals with cognitive challenges should take time and should not be seen as a single process. ${ }^{38}$ Recruitment protocols therefore require critical reflection around ways that a singular focus on targets and timeliness may be contrary to an ethically grounded approach in the application of consent tools. One strategy here may be to base recruitment rates on studies that have applied iterative learning during consent, or to implement a stepped approach, initially setting low recruitment targets which gradually increase as recruiters' skills and confidence improve.

Third, the effectiveness of consent tools used with the intent of educating to include is highly dependent on the interpersonal skills of the recruiter. Research has demonstrated the influential role that recruiters play in consent rates ${ }^{34}$ with enthusiasm and clear communication style demonstrating a powerful facilitative role in encouraging individuals with intellectual disability to engage in research. ${ }^{38}$ Implicit attitudes and beliefs also matter in such contexts, where stigmatising beliefs that persons with cognitive challenges are unable to consent can impact and influence the consenting process. Ultimately, evidence suggests that participation in research does matter to persons with mental disorder and intellectual disability; however, establishing trust with recruiters and researchers is vitally important ${ }^{10}$ in order to potentially mitigate the reluctance and suspicion of families and carers about research involving such individuals. ${ }^{38}$ As a result, recruiter training should focus on rapport building including an awareness of stigmatising attitudes towards individuals with intellectual disability and mental disorders, as well as a better understanding of the relationship dynamics shared with carers and family members. It would make strategic sense then to collaborate with people with intellectual disability and mental disorders in the planning and implementation of recruiter training.

\section{Substantive implications}

This educative approach raises difficult questions around whether a participatory ethos towards persons with mental disorder and intellectual disability can be coherently aligned with the current normative aspirations and framing of psychiatric genomics research. This issue goes beyond the purpose of 
this paper; however, some cursory remarks around two areas of concern are worthwhile. First is the question of what substantive participation and representation of such persons really means. Research models which are thought best to reflect this shift in orientation include qualitative studies that critically situate researchers and their positioning vis-à-vis participants and seek creative strategies to better capture and understand the subjective voice, perceptions and experiences of individuals living with mental disorder and intellectual disability. Moreover, meaningful participation is equated with not just participation, but the inclusion of such individuals in setting research agendas, the allocation of resources and participation in ethical review boards. ${ }^{11}$ Community engagement through mechanisms such as community advisory boards is one consideration ${ }^{39}$, although there is much debate about how to frame the goals and implementation of such mechanisms, and evaluate their effectiveness in meaningful ways. ${ }^{40}$

How clinical research into psychiatric genomics can encompass more meaningful forms of participation remains unclear, not just because of the research models typically used in the clinical context, but in large part because of intractable tensions around normative intent. Underlying the participatory ethos of the supported decision-making paradigm is a strong commitment to the accommodation of mind and bodily difference, with a focus on altering environmental and structural conditions which function as barriers to the equal treatment of persons with cognitive impairments. The aspiration, in other words, is to broaden societal and cultural understandings around cognitive challenges, to use research to change society in ways that are more accepting and supportive of those with intellectual disability and mental disorder.

By contrast, psychiatric genomics research is firmly committed to the framework of early intervention and the eventual prevention of certain neurodevelopmental/mental disorders, where improved understanding of the genetic architecture underlying such conditions may pave the way for targeted druggable interventions. ${ }^{41}$ Starkly put, the application of consent tools as educative mechanisms designed to include and foster participation in psychiatric genomics studies appears fundamentally contradictory, given that this inclusive ethos is applied to research that presupposes participants' conditions are undesirable and worthy of eliminating. This brings into sharp relief the challenge of balancing the interests of psychiatric genomics researchers and participants, as well as the urgent need for further critical exploration of the ways in which the impetus towards early intervention/prevention strategies in psychiatric genomics research can be meaningfully reconciled with the equal and respectful treatment that is owed to persons with mental disorder and intellectual disability.

\section{CONCLUSION}

Our view is that even large-scale psychiatric genomics studies need to ensure that recruitment and consent tools accord with the participatory/supported decision-making paradigm within patient-centred psychiatry and human rights instruments, like the CRPD. Used as educational tools, the consenting process and its constituent skills of hermeneutic competence have the potential to help realise the aims of supported decision-making and improve the interactions between researchers and potentially vulnerable participants with mental disorder and intellectual disability. These skills are ethically valuable and applicable, not just in psychiatric genomics studies, but in human research fields more generally, whether from a medical or social scientific focus, and particularly in studies where complex research concepts are at play. By investing time and resources, persons of different cognitive abilities may be empowered to take a degree of risk in making their decisions and act as co-producers of knowledge about their lives and experiences. Moreover, this supportive, educative framework for consenting tools may be especially relevant to more communitarian cultural traditions, where decision-making is often a collective process embedded within the relational and social context.

Future investigations nonetheless need to explore the practicalities of this framework in cases of profound cognitive and communicative challenges. While we have not made the argument here, our view gestures towards a more spectrum view of capacity ${ }^{27}$ which nonetheless prioritises the overarching normative ethos of the CRPD—something akin to the emphasis on the person's wishes, feelings and values in some best interests decision-making frameworks (eg, the Mental Capacity 2005 in England and Wales). As such, any introduction of some form of substituted decision-making mechanisms must be a last resort following repeated efforts to support potential consent/ non-consent through iterative learning, keeping always in mind, however, the possibilities of relational dynamics, coercion, and exploitation. We lack the space to flesh out this point here but suffice to say that the difficulty in specifying a concrete threshold is itself a clear manifestation of the perpetual challenge to achieve an ethically appropriate balance between the safeguarding and protection of persons with mental disorder and intellectual disability.

Also warranting future discussion is the even more pressing question of whether recalibrating the consenting process can sufficiently mitigate worries around the preventative/early intervention agenda of psychiatric genomics, specifically whether such an agenda may be at odds with the respect and acceptance of persons with cognitive impairments which grounds the supported decision-making paradigm. Our suggestion is that the conceptual shift in informed consent processes-from screening to exclude, to educating to be more inclusive-should be viewed as an initial step in unpacking this much larger issue. The next step may be more genuine and meaningful community engagement efforts that enable persons with mental disorders and intellectual disabilities to express their concerns, shape and enrich the research and clinical agenda in psychiatric genomics.

Acknowledgements We would like to acknowledge the African Ethics Working Group of NeuroGenE for their feedback on an earlier draft of the paper, as well as the helpful comments of two anonymous reviewers.

Contributors All three authors contributed to the conceptualisation and first draft of the paper. CK and MC were responsible for subsequent drafts and edits to the paper.

Funding Funding for the research of the paper was provided by the NeuroGenE project, funded by the Broad Institute of MIT and Harvard.

Competing interests None declared.

Patient consent for publication Not required.

Provenance and peer review Not commissioned; externally peer reviewed.

\section{ORCID iD}

Camillia Kong http://orcid.org/0000-0002-0551-0689

\section{REFERENCES}

1 de Vries J, Bull SJ, Doumbo 0, et al. Ethical issues in human genomics research in developing countries. BMC Med Ethics 2011;12(1):5.

2 Afolabi MO, Okebe JU, McGrath N, et al. Informed consent comprehension in African research settings. Trop Med Int Health 2014;19(6):625-42.

3 Campbell MM, Susser E, Mall S, et al. Using iterative learning to improve understanding during the informed consent process in a South African psychiatric genomics study. PLoS One 2017;12(11):e0188466. 
4 Appelbaum PS, Grisso T. Macarthur competence assessment tool for clinical research (MacCAT-CR).. Professional Resource Press/Professional Resource Exchange, 2001.

5 Jeste DV, Palmer BW, Appelbaum PS, et al. A new brief instrument for assessing decisional capacity for clinical research. Arch Gen Psychiatry 2007:64(8):966-74.

6 Kowal EE. Genetic research in Indigenous health: significant progress, substantial challenges. Med J Aust 2012;197(1):19.

7 United nations convention on the rights of persons with disabilities.

8 lacono T, Carling-Jenkins R. The human rights context for ethical requirements for involving people with intellectual disability in medical research. J Intellect Disabil Res 2012;56(11):1122-32

9 Mezzich JE. World psychiatric association perspectives on person-centered psychiatry and medicine. Int J Integr Care 2010;10(5).

10 McDonald KE, Kidney CA, Patka M. 'You need to let your voice be heard': research participants' views on research. J Intellect Disabil Res 2013;57(3):216-25.

11 McKenzie J, Mii G, Gcaza S. With or without us? an audit of disability research in the southern African region. AJOD 2014;3(2).

12 Gooding P. Supported decision-making: a rights-based disability concept and its implications for mental health law. Psychiatry, Psychology and Law 2013;20(3):431-51.

13 Lennard C. Fluctuating capacity and impulsiveness in acquired brain injury: the dilemma of "unwise" decisions under the Mental Capacity Act. The Journal of Adult Protection 2016;18(4):229-39.

14 Capacity TT. Mental mechanisms, and unwise decisions. Philos Psychiatr Psychol 2011;18(2):127-32.

15 Slade M, Adams N, O'Hagan M. Recovery: past progress and future challenges. International Review of Psychiatry 2012:24(1):1-4.

16 Oliver M. Understanding Disability: From Theory to Practice (second edition). Palgrave Macmillan 2009

17 United Nations Committee on the Rights of Persons with Disabilities. General Comment No. 1-Article 12: Equal recognition before the law, UN doc. No. CRPD/C/ GC/1, adopted at the 11th Session 2014

18 Szmukler G. Compulsion and "coercion" in mental health care. World Psychiatry 2015; 14(3):259-61.

19 Freeman MC, Kolappa K, de Almeida JMC, et al. Reversing hard won victories in the name of human rights: a critique of the General Comment on Article 12 of the UN Convention on the Rights of Persons with Disabilities. The Lancet Psychiatry 2015:2(9):844-50.

20 Kong C. The Convention for the Rights of Persons with Disabilities and Article 12: Prospective feminist lessons against the "will and preferences" paradigm. Laws 2015:4(4):709-28.

21 Mji G, Schneider M, Vergunst R, et al. On the ethics of being photographed in research in rural South Africa: views of people with disabilities. Disabil Soc 2014;29(5):714-23.

22 Jurkowski JM. Photovoice as participatory action research tool for engaging people with intellectual disabilities in research and program development. Intellect Dev Disabil 2008;46(1):1-11
23 Taua C, Neville C, Hepworth J. Research participation by people with intellectual disability and mental health issues: an examination of the processes of consent. Int J Ment Health Nurs 2014;23(6):513-24.

24 Cameron L, Murphy J. Obtaining consent to participate in research: the issues involved in including people with a range of learning and communication disabilities. Br J Learning Disab 2007;35(2):113-20.

25 Beail N. Williams K. Using qualitative methods in research with people who have intellectual disabilities. J App/ Res Intellect Disabil 2014;27(2):85-96.

26 Knox M, Mok M, Parmenter TR. Working with the experts: collaborative research with people with an intellectual disability. Disabil Soc 2000;15(1):49-61.

27 Kong C. Mental Capacity in relationship: Decision-making, dialogue, and autonomy. Cambridge University Press 2017.

28 Kong C, Ruck Keene A. Overcoming challenges in the Mental Capacity Act 2005 Practical guidance for working with complex issues. Jessica Kingsley Publishers, 2018

29 Dreyfus HL. Skillful coping: Essays on the phenomenology of everyday perception and action. Oxford University Press, 2014

30 Menkiti IA. Person and community in African traditional thought. African philosophy: An introduction 1984;3:171-82.

31 Gyekye K. Tradition and modernity: philosophical reflections on the African experience. ord University Press 1997.

32 Gyekye K. An essay on African philosophical thought: The Akan conceptual scheme. Temple University Press, 1995.

33 Douglass, A. Mental capacity: Updating New Zealand's law and practice. (Report for the New Zealand Law Foundation, Dunedin, 2016. Available: http://www. alisondouglass.co.nz.

34 Campbell MM, de Vries J, Mqulwana SG, et al. Predictors of consent to cell line creation and immortalisation in a South African schizophrenia genomics study. BMC Med Ethics 2018:19(1):72

35 Mandava A, Pace C, Campbell B, et al. The quality of informed consent: mapping the landscape. A review of empirical data from developing and developed countries. J Med Ethics 2012;38(6):356-65.

36 Gadamer HG. Truth and method. Weinsheimer J, Marshall DG, eds. USA: Bloomsbury Publishing, 2004.

37 Gilbert T, Bosquet A, Thomas-Antérion C, et al. Assessing capacity to consent for research in cognitively impaired older patients. Clin Interv Aging 2017:12:1553-63.

38 Nicholson L, Colyer M, Cooper S-A. Recruitment to intellectual disability research: a qualitative study. J Intellect Disabil Res 2013;57(7):647-56.

39 Campbell MM, Susser E, de Vries J, et al. Exploring researchers' experiences of working with a researcher-driven, population-specific community advisory board in a South African schizophrenia genomics study. BMC Med Ethics 2015;16(1):45.

40 Tindana P, Campbell M, Marshall P, et al. Developing the science and methods of community engagement for genomic research and biobanking in Africa. Glob. Health Epidemiol. 2017:2.

41 Insel TR, Collins FS. Psychiatry in the genomics era. AJP 2003;160(4):616-20. 